\title{
DESENVOLVIMENTO DE UMA CENTRÍFUGA PARA SOLIDIFICAÇÃO DE MATERIAIS EM ALTAS ACELERAÇÕES*
}

\author{
Plínio Ivo Gama Tenório ${ }^{1}$ \\ Chen Ying $A n^{2}$ \\ Rafael Cardoso Toledo 3 \\ Irajá Newton Bandeira ${ }^{4}$ \\ Filipe Estevão Freitas ${ }^{4}$ \\ Edson Fernando Fumachit \\ Pedro Tiago Bernabé Lomas ${ }^{4}$
}

\section{Resumo}

Diferentes gravidades, maiores ou menores que a terrestre, influenciam no processo de solidificação dos materiais. A fim de estudar esses efeitos, em ligas metálicas e semicondutores, iniciou-se o desenvolvimento de uma centrífuga no Laboratório Associado de Sensores e Materiais do Instituto Nacional de Pesquisas Espaciais (LAS/INPE). O objetivo do equipamento é simular altas acelerações durante as fases da solidificação de materiais e simular as condições de lançamento do foguete VSB30 , veículo que atinge cerca de 180 RPM (rotações por minuto) durante o voo ascendente, utilizado para realização de experimentos em ambiente de microgravidade. Assim, esse trabalho apresentará o projeto, a fabricação de componentes e a montagem da centrífuga para estudo da influência da aceleração da gravidade na solidificação de materiais.

Palavras-chave: Centrifuga; Solidificação; Alta gravidade.

\section{DEVELOPMENT OF A CENTRIFUGE FOR MATERIALS PROCESSING IN HIGH ACCELERATIONS}

\section{Abstract}

Different gravity, bigger or smaller than the terrestrial, influence the solidification process of the materials. To study these effects, in metal alloys and semiconductors, the development of a centrifuge was initiated in the Associated Laboratory of Sensors and Materials of the National Institute for Space Research (LAS / INPE). The objective of equipment is simulate high accelerations during the materials solidification phases and simulate the launch conditions of the VSB-30, a launcher that during the upward flight reaches about 180 RPM (revolutions per minute), which was used in the Maracati II operation, where laboratory experiments were performed. So, this work will present the design, manufacture of components and assembly of equipment and its use, in view of the objectives, restrictions and security requirements imposed on it.

Keywords: Centrifuge; Solidification; High gravity.

1 Tecnólogo em Manufatura Aeronáutica, aluno de mestrado, Laboratório Associado de Sensores e Materiais, Instituto Nacional de Pesquisas Espaciais, São José dos Campos, São Paulo, Brasil.

2 Doutor em Engenharia Aeronáutica e Mecânica, Laboratório Associado de Sensores e Materiais, Instituto Nacional de Pesquisas Espaciais, São José dos Campos, São Paulo, Brasil.

3 Doutor em Engenharia e Tecnologia Espaciais, Laboratório Associado de Sensores e Materiais, Instituto Nacional de Pesquisas Espaciais, São José dos Campos, São Paulo, Brasil.

4 Laboratório Associado de Sensores e Materiais, Instituto Nacional de Pesquisas Espaciais, São José dos Campos, São Paulo, Brasil. 


\section{INTRODUÇÃO}

Altas acelerações podem afetar os processos de nucleação e crescimento de grão durante a solidificação de materiais, sendo capaz de alterar a transferência de massa e calor e, devido à ação da aceleração de Coriolis no fluído, a estabilidade do fluxo [1]. Como esses efeitos alteram a microestrutura do material [2], podem-se obter comportamentos diferentes em determinadas solicitações, se comparando com métodos convencionais de fundição e resfriamento [3 e 4].

Em geral os equipamentos utilizados nesses estudos são centrífugas [5 e 6], já que incluindo a força centrípeta no sistema combinada com a força peso, resulta em acelerações $(\vec{g})$ maiores que a da gravidade da Terra [7].

Sendo assim, o objetivo desse trabalho é desenvolver um equipamento capaz de simular condições de altas acelerações com rotações de até 180 RPM (rotações) para solidificação de metais e semicondutores com ponto de fusão de até $1000^{\circ} \mathrm{C}$ [8]. O equipamento também possibilita simular as condições de lançamento do foguete VSB-30, veículo sub-orbital desenvolvido pelo Instituto de Aeronáutica e Espaço do Departamento de Ciência e Tecnologia Aeroespacial (IAE/DCTA), utilizado pelo programa Microgravidade da Agência Espacial Brasileira (AEB) para realização de experiências científicas em ambiente de microgravidade [9,10 e 11].

\section{FÍSICA DA CENTRÍFUGA}

Quando uma massa na Terra é submetida a uma velocidade angular ( $\omega)$ e está deslocada do eixo de rotação é inserida uma força no sistema, chamada de centrípeta $\left(\overrightarrow{F_{c}}\right)$ [12]. Quando observada a partir de um referencial podemos chama-la de centrifuga $\left(\overrightarrow{C_{p}}\right)$ com mesmo modulo e direção da $\overrightarrow{F_{c}}$ mas com sentido contrario [7]. No equipamento o referencial adotado foi o forno, que sofria, principalmente, o efeito de três forças: tração $(\vec{T})$, peso $(\vec{P})$ e a centrifuga (Figura 1).

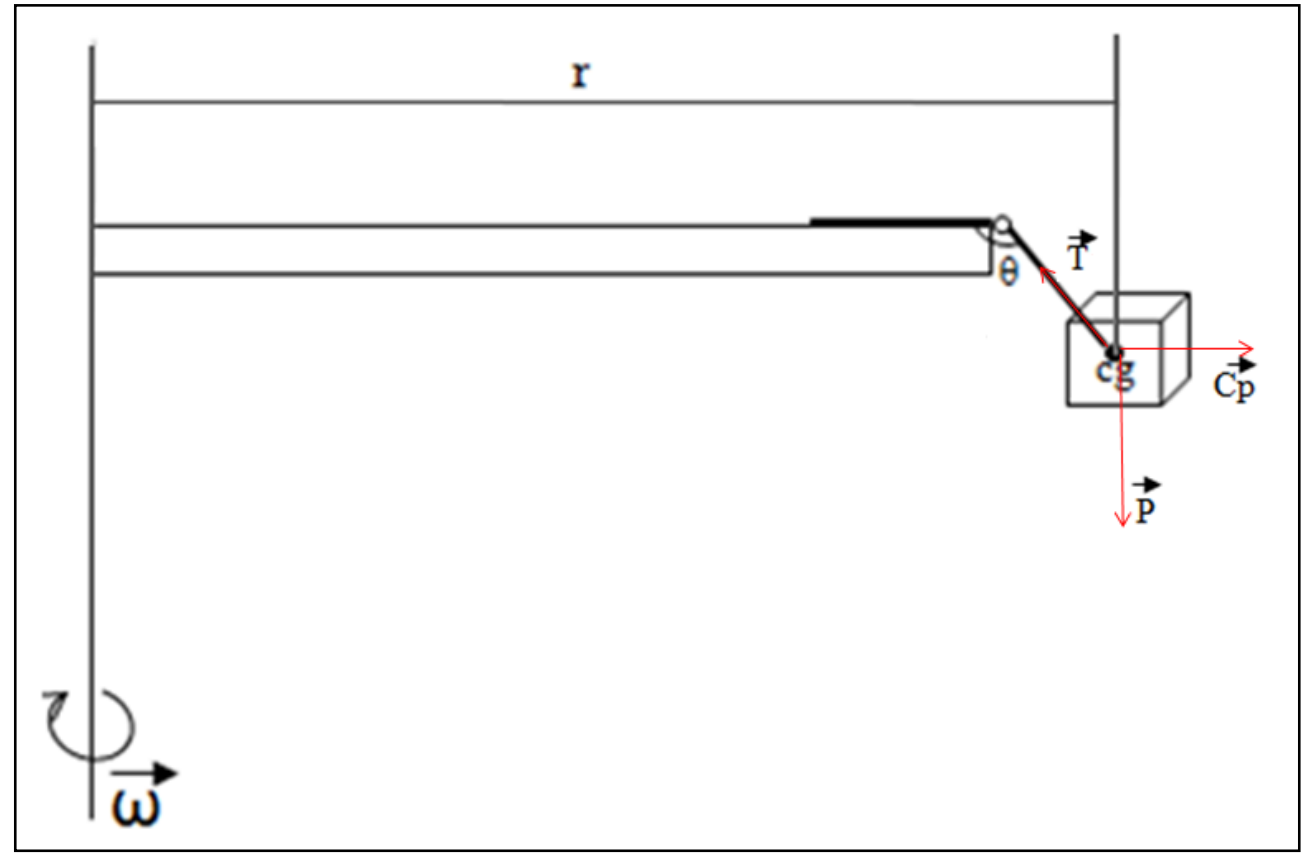

Figura 1. Esquema de forças na centrífuga. 


\section{MATERIAIS E MÉTODOS}

O desenvolvimento do equipamento baseou-se no conceito desenvolvido por An et al. [1] e nas principais necessidades do laboratório, estas foram elencadas em: (i) restrições geométricas e de segurança; (ii) demanda de estudos e pesquisa; e (iii) a disponibilidade e/ou facilidade de adquirir os materiais e os métodos de fabricação. O projeto da centrífuga foi divido em duas aplicações, solidificação materiais e simulação de rotações, e cinco submontagens, são essas: estrutura metálica, motor e contato elétrico rotativo estes comuns em ambas às aplicações/ensaios e braço, forno, controlador de temperatura e sistema de aquisição de dados específicos para cada aplicação/ensaio.

\subsection{Estrutura Metálica}

A estrutura metálica (Figura 2) serve como suporte do sistema rotativo, sendo assim, é importante garantir a sua segurança e eficiência estrutural, a fim de melhorar o funcionamento e a durabilidade do equipamento [13].
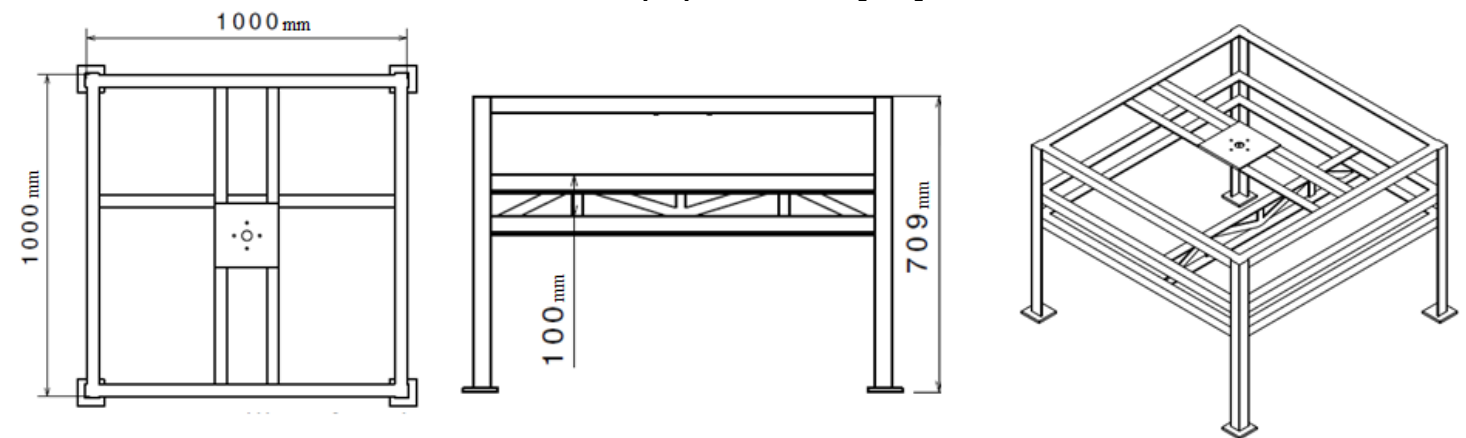

Figura 2. Desenho e principais cotas da estrutura metálica.

\subsection{Motor}

Para que a aceleração resultante seja significativamente maior que $\vec{g}$ são necessários dois conjuntos: o motor e o braço, uma vez que a $\vec{a}_{\text {centípeta }}$ é uma grandeza vetorial dependente da velocidade e do raio de uma circunferência [7]. $O$ modelo de motor escolhido foi o W22 plus da WEG®, os atributos levados em consideração foram a melhor precisão no controle de velocidade, número de polos e ter baixos níveis de ruído e vibração [14]. Para o seu acionamento foi instalado um inversor de frequência da mesma fabricante, modelo CFW-08, que possibilita maior durabilidade para o equipamento, além de facilitar a utilização para qualquer operador [15].

\subsection{Contato Elétrico Rotativo}

A fim de transmitir a rotação, com mínima perda, do motor para o braço e auxiliar na aquisição de dados foi desenvolvido um contato elétrico rotativo (Figura 3). Sua montagem consiste basicamente em três rolamentos, sendo um de apoio, um eixo e o contato elétrico separados por um material isolante [1 e 8]. 

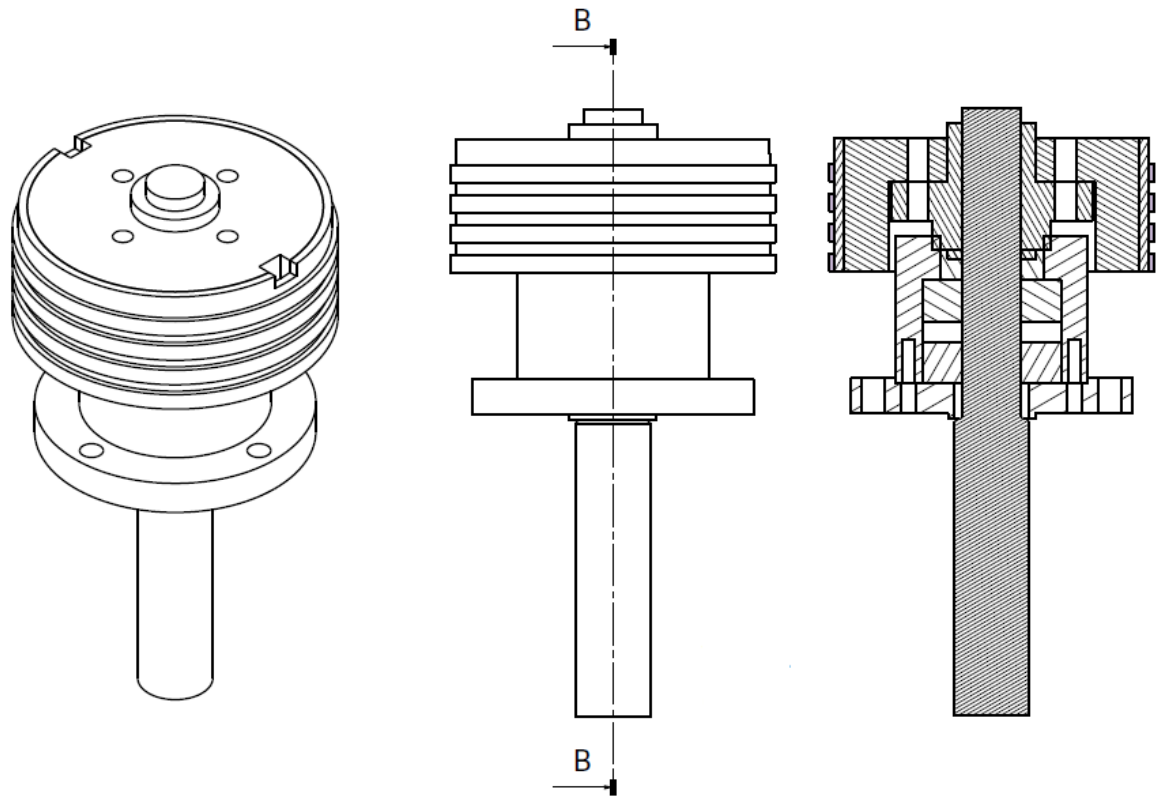

Figura 3. Desenho do contato elétrico rotativo.

\subsection{Braço}

Cada ensaio demanda um braço especifico (Figura 4), por que, essa peça serve de suporte e tem a função de deslocar o forno em relação ao eixo do motor, uma vez que, para que seja inserida a força centrípeta no sistema é necessário que se tenha uma distância (raio) entre o referencial inercial e o centro da rotação [12].

(a)

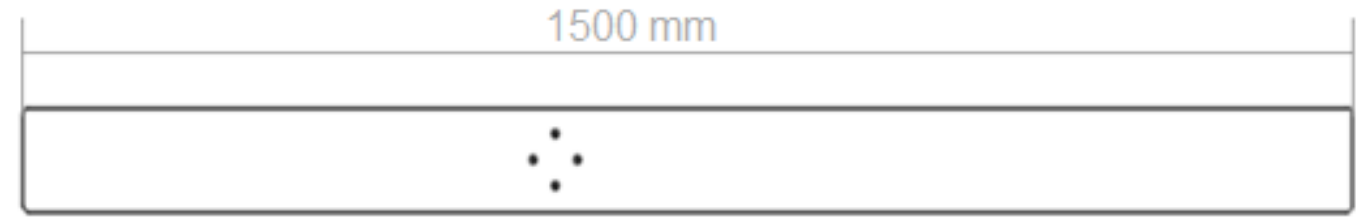

(b)

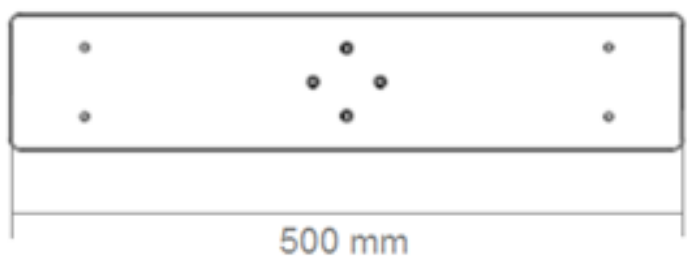

Figura 4. Braços a) ensaio de solidificação; b) simulação de rotações.

\subsection{Forno}

Assim como no caso dos braços, existe a necessidade de um forno especifico para cada tipo de ensaio (Figura 5). O utilizado para simulação das rotações do VSB-30 é exatamente o mesmo utilizado na Operação Maracati II [10 e 16]. Ambos são semelhantes, consistem de um cilindro, que gera calor através da tensão que passa em uma resistência fixada em uma de suas extremidades. O compartimento de carga é tubular, projetado para o encaixe de ampolas de quartzo contendo o material a ser fundido, sendo completamente isolado termicamente, a fim de maximizar sua eficiência térmica [16 e 17]. 
(a)

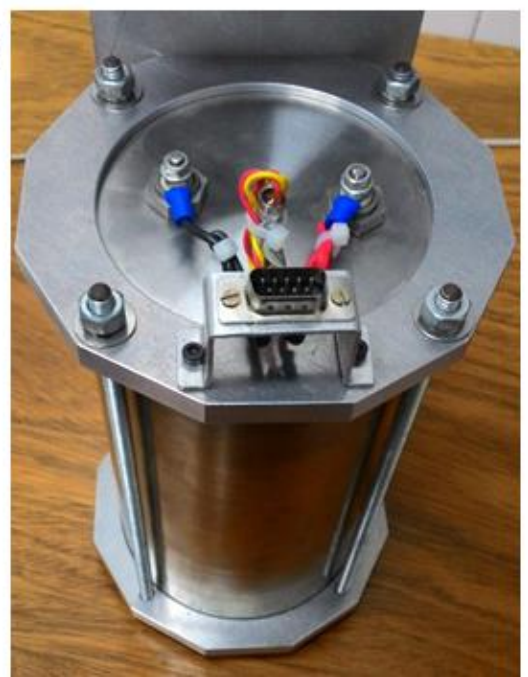

(b)

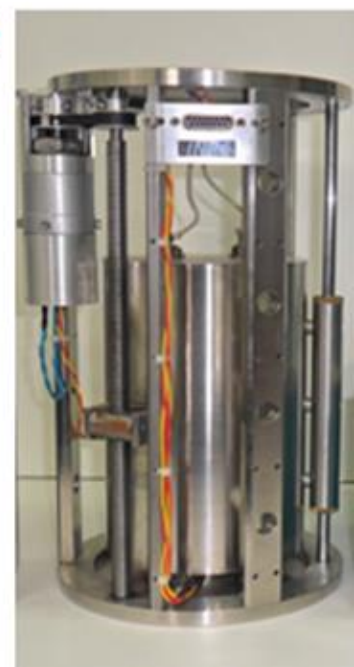

Figura 5. (a) Forno para ensaios de solidificação; (b) Forno da Operação Maracati II [17].

\subsection{Controlador}

Cada forno utiliza um controlador especifico (Figura 6). Sua função é acionamento e controle da temperatura. Nas simulações de rotação do VSB-30, será utilizado o mesmo dos experimentos anteriores. Para os ensaios em altas acelerações foi desenvolvido um protótipo, que possui dois circuitos diferentes: um para controle (com corrente e potência menor) e outro para o fornecer a energia necessária para 0 aquecimento do forno (com corrente e potência maior).

(a)

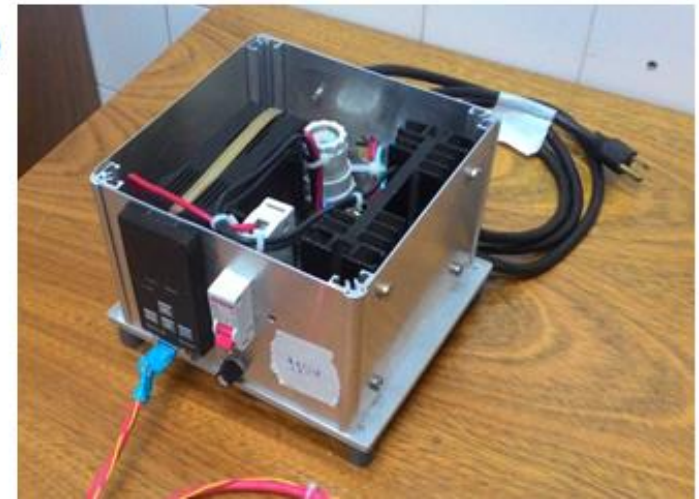

(b)

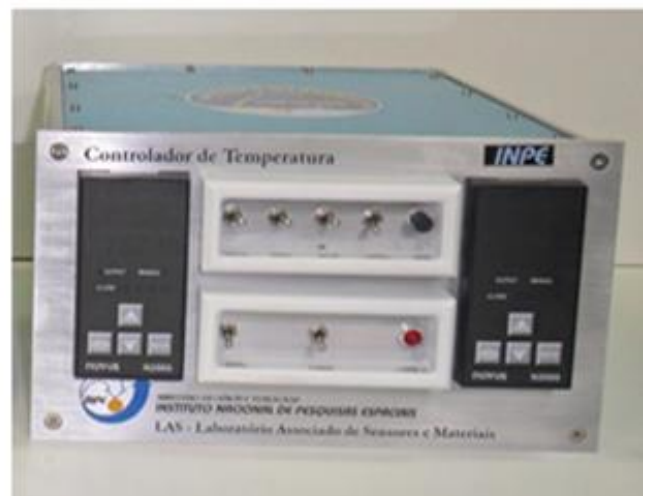

Figura 6. Controlador (a) ensaios em altas acelerações; (b) Operação Maracati II [16].

\section{RESULTADOS E DISCUSSÃO}

\subsection{Base}


A base é uma estrutura comum para os dois experimentos (Figura 7). Consiste da estrutura metálica e do contato elétrico rotativo.

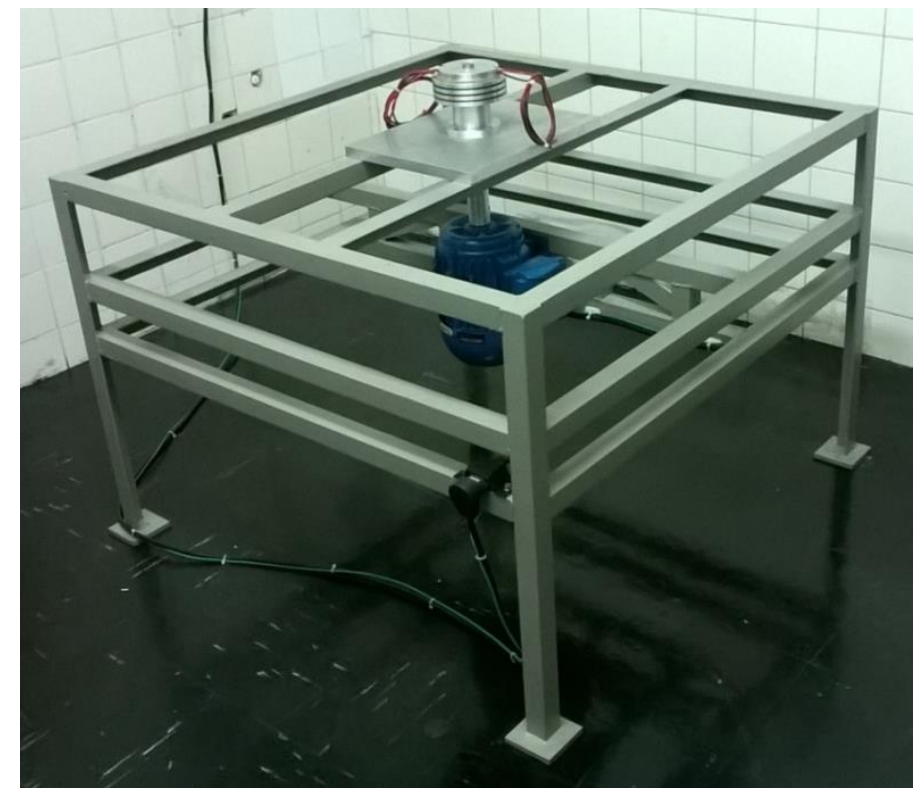

Figura 7. Base comum do equipamento.

A estrutura metálica foi simulada em elementos finitos, antes da fabricação, os resultados apresentaram deformação da ordem de $10^{-2}$ no local de maior solicitação, com fator de segurança 1,5, sendo assim, o projeto da estrutura metálica foi considerado adequado para a aplicação (Figura 9) [13]. O material escolhido foi o aço ASTM A36 e sua manufatura consistiu no processo de soldagem de barras.
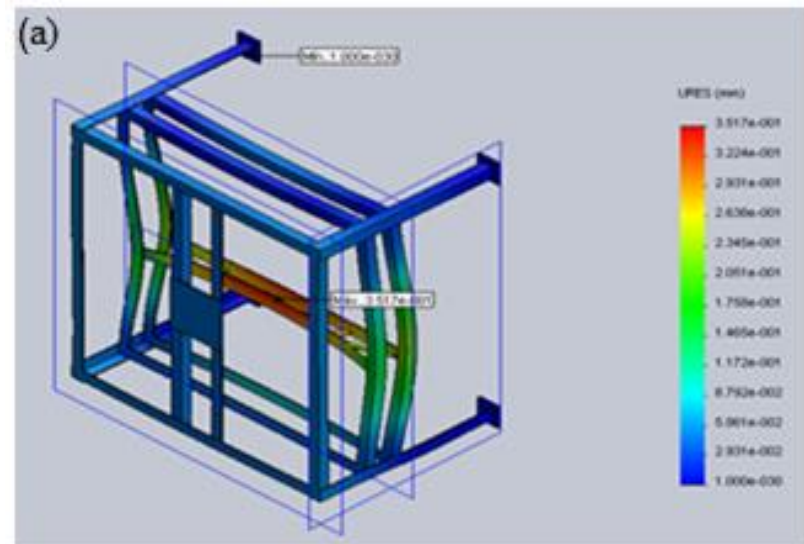

(b)

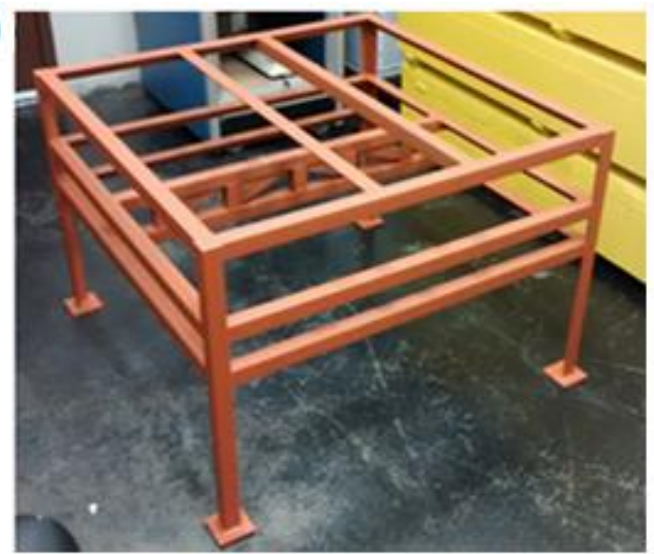

Figura 5. a) Simulação em elementos finitos; b) Estrutura Metálica

A montagem do contato elétrico rotativo (Figura 9) sofrera, principalmente, carga compressiva, devido a isso, foram escolhidos materiais dúcteis para as peças, alumínio AA 6351-T6 e PVC (policloreto de vinila), sendo o segundo utilizado somente para isolamento elétrico dos anéis de contato, sem desempenhar qualquer função estrutural. Os processos de fabricação envolvidos foram usinagem e fresamento. 

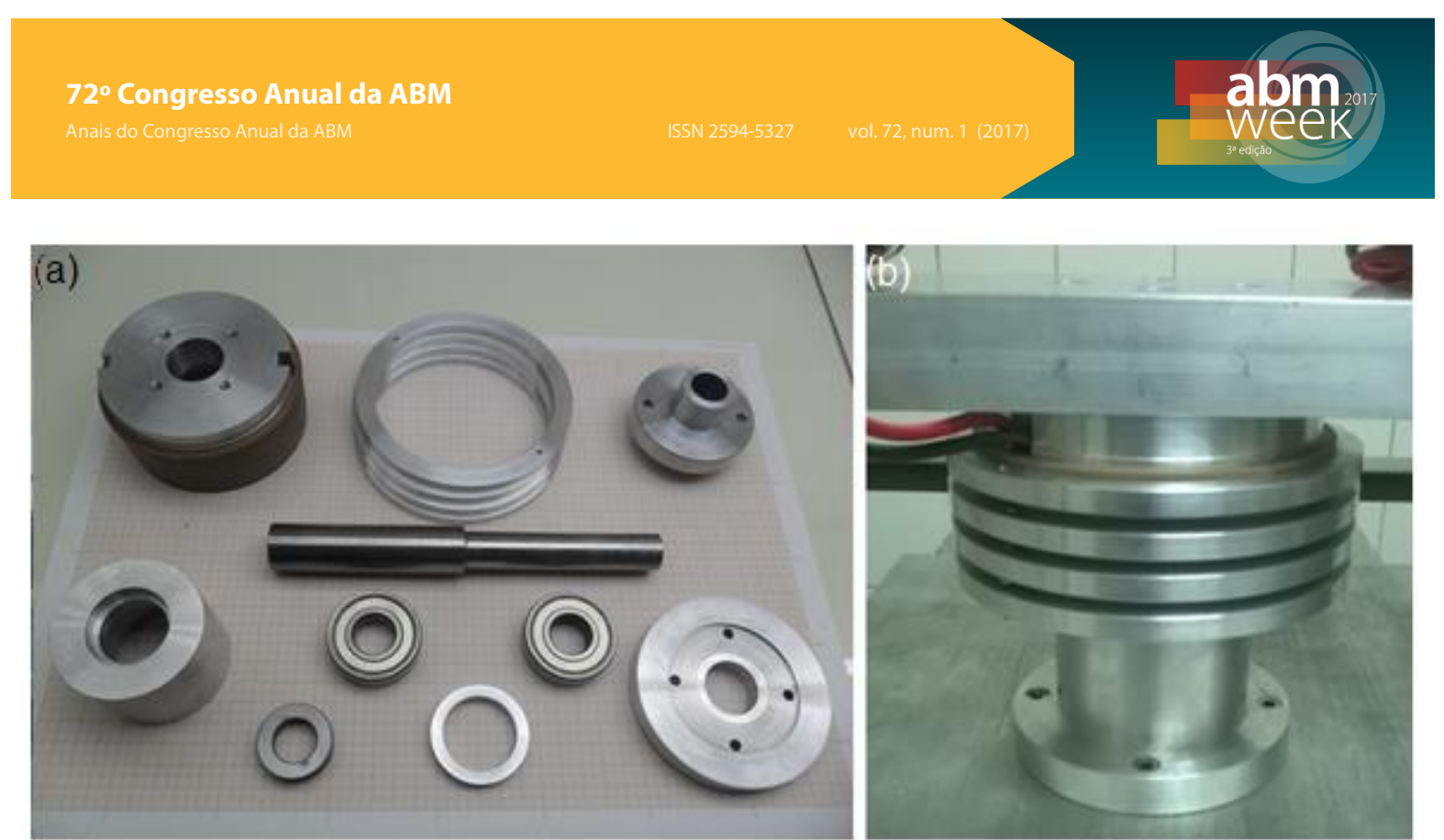

Figura 6. a) peças do contato elétrico rotativo; b) montagem instalada.

\subsection{Ensaios em altas acelerações}

A montagem do equipamento utilizado nos ensaios em altas acelerações (Figura 11), consiste em fixar o forno ao braço através de uma dobradiça (Figura 12), que possui dois rolamentos e suporta até $45 \mathrm{~kg}$, essa fixação garante alinhar o material ensaiado, o mais próximo possível, com o vetor da força resultante no sistema, a fim de facilitar os cálculos para determinação do $\vec{g}$ na amostra.

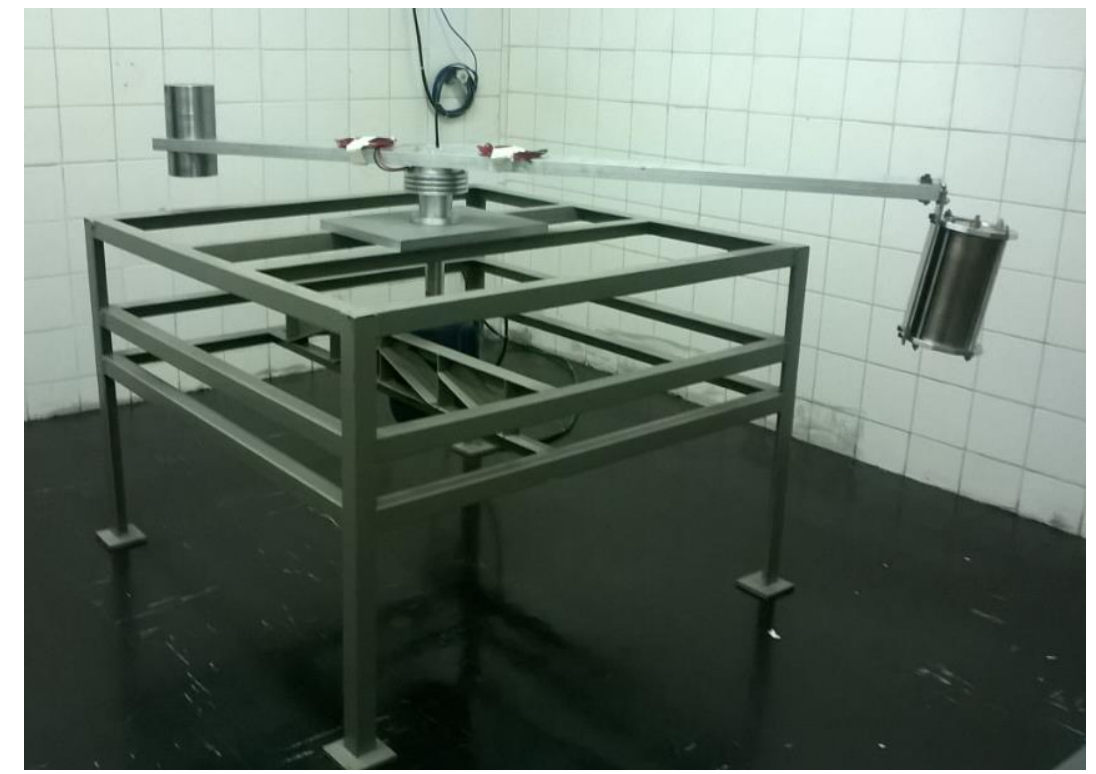

Figura 7. Equipamento para ensaio em altas acelerações.

Esse deslocamento de massa, ao longo do tempo de utilização da centrifuga, pode gerar desbalanceamento no motor e, por consequência, causar vibração no equipamento, para evitar isso, contrapesos cilíndricos foram elaborados para serem instalados na extremidade oposta, a fim de garantir que o centro de gravidade ficasse alinhado com o do eixo do motor [18]. 


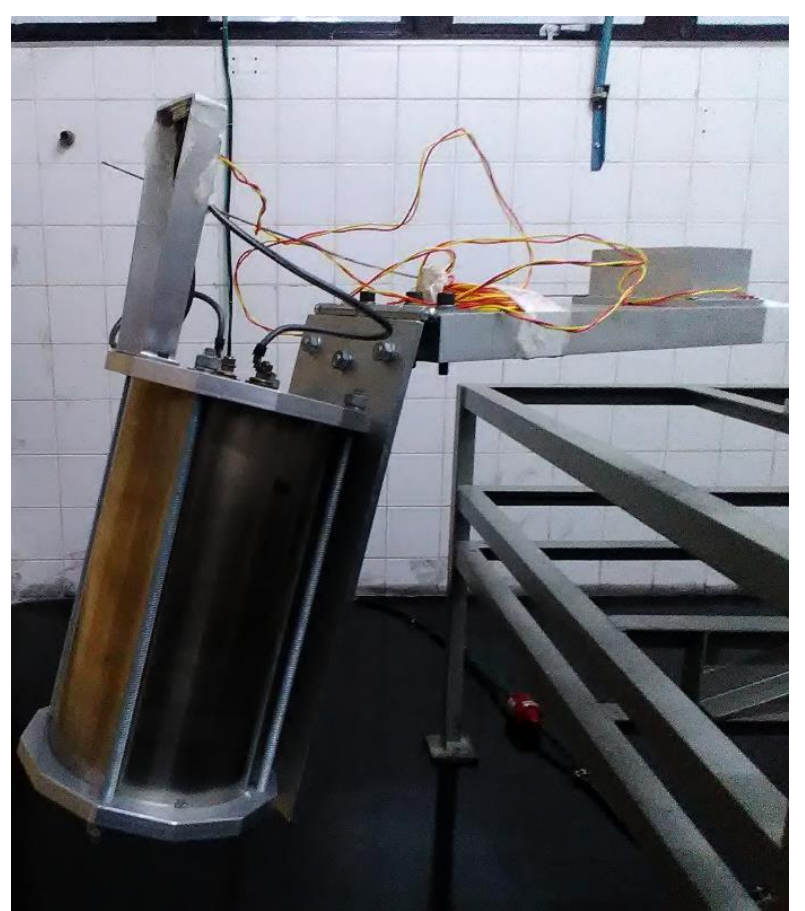

Figura 8. Fixação do forno no braço para ensaios com altas acelerações.

A determinação da quantidade de massa e posição dos contrapesos (Figura 13) que seria necessária para balancear o sistema partiu do princípio de equilíbrio estático (Equação 1), no qual a somatória de momentos do sistema deve ser igualada a zero [18].

$$
\mathrm{m}_{\text {contrapeso }}=\frac{\mathrm{m}_{\text {forno }}+\mathrm{b}_{\text {forno }}}{\mathrm{b}_{\text {contrapeso }}}
$$

Onde a mforno é $4 \mathrm{~kg}$, o b borno é $0,9 \mathrm{~m}$ e o bcontrapeso é $0,6 \mathrm{~m}$.

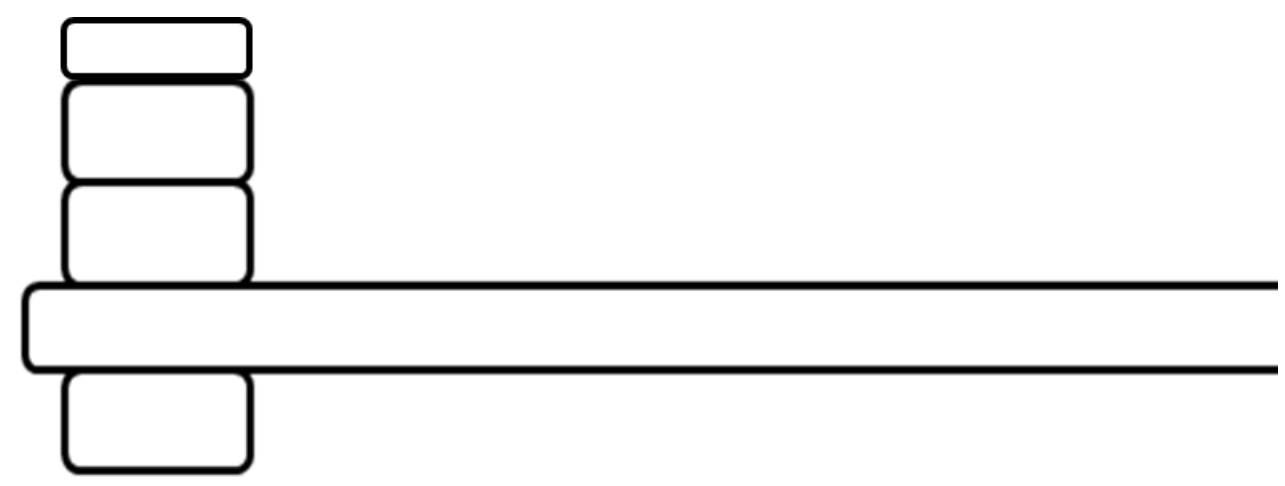

Figura 13. Sistema de balanceamento do braço.

Um fator importante para o ensaio é conhecer a convecção do calor dentro forno. Esse dado pode otimizar vários parâmetros, como por exemplo, no tempo de experimento, uma vez que, posicionando a amostra na posição mais quente do compartimento de carga, se garantirá uma fundição mais rápida.

Para isso foi determinado experimentalmente o perfil de temperatura do forno (Figura 14), considerando o braço como referência para a angulação, com 
temperaturas de controle (Tset) de $200^{\circ} \mathrm{C}$ e $300^{\circ} \mathrm{C}$ e estático. Também foi determinado o perfil dinâmico (Figura 15), estado do forno durante a solidificação, com as mesmas temperaturas de controle do anterior e com rotação de 60RPM.

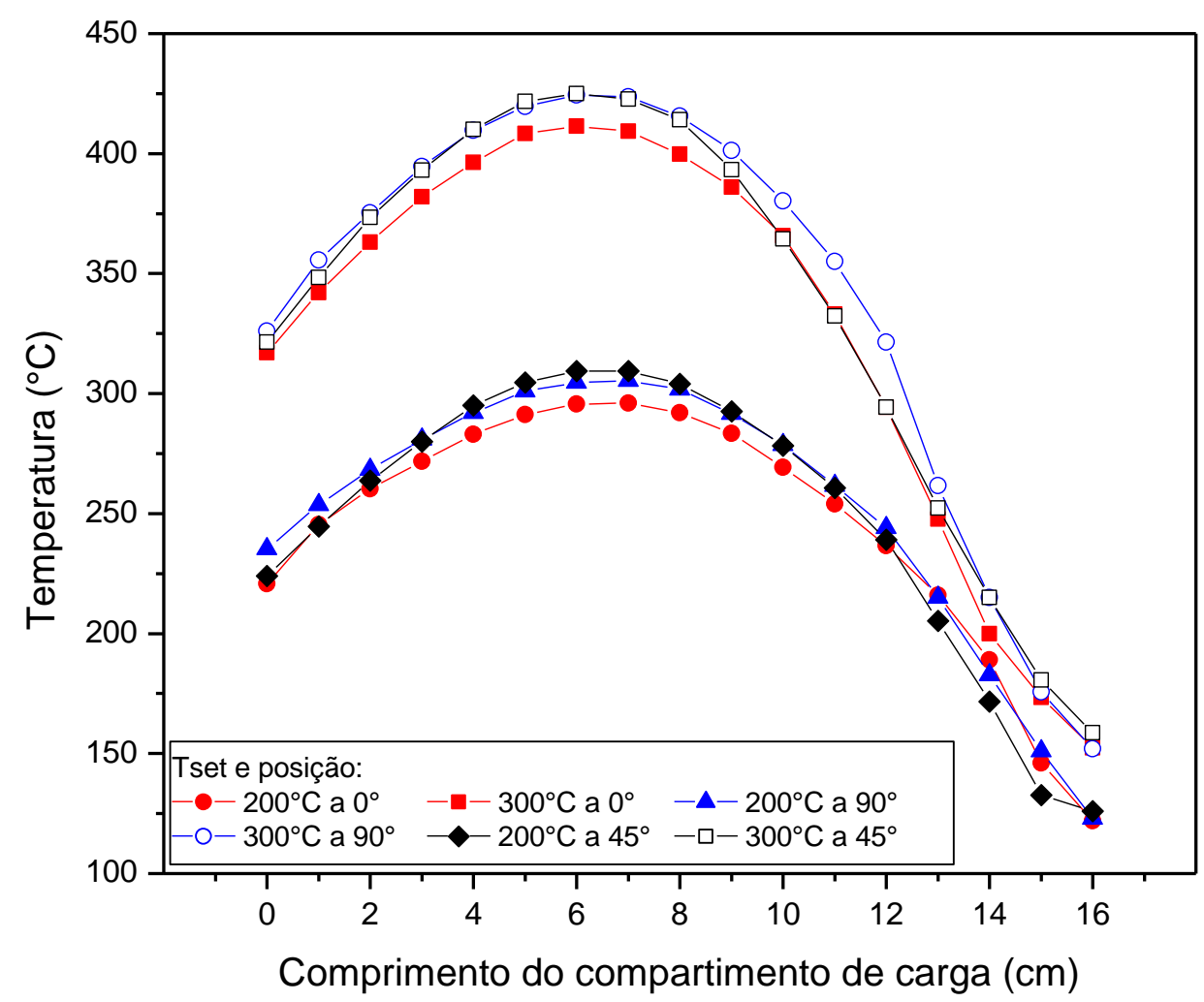

Figura 9. Perfil de temperatura do forno estático.

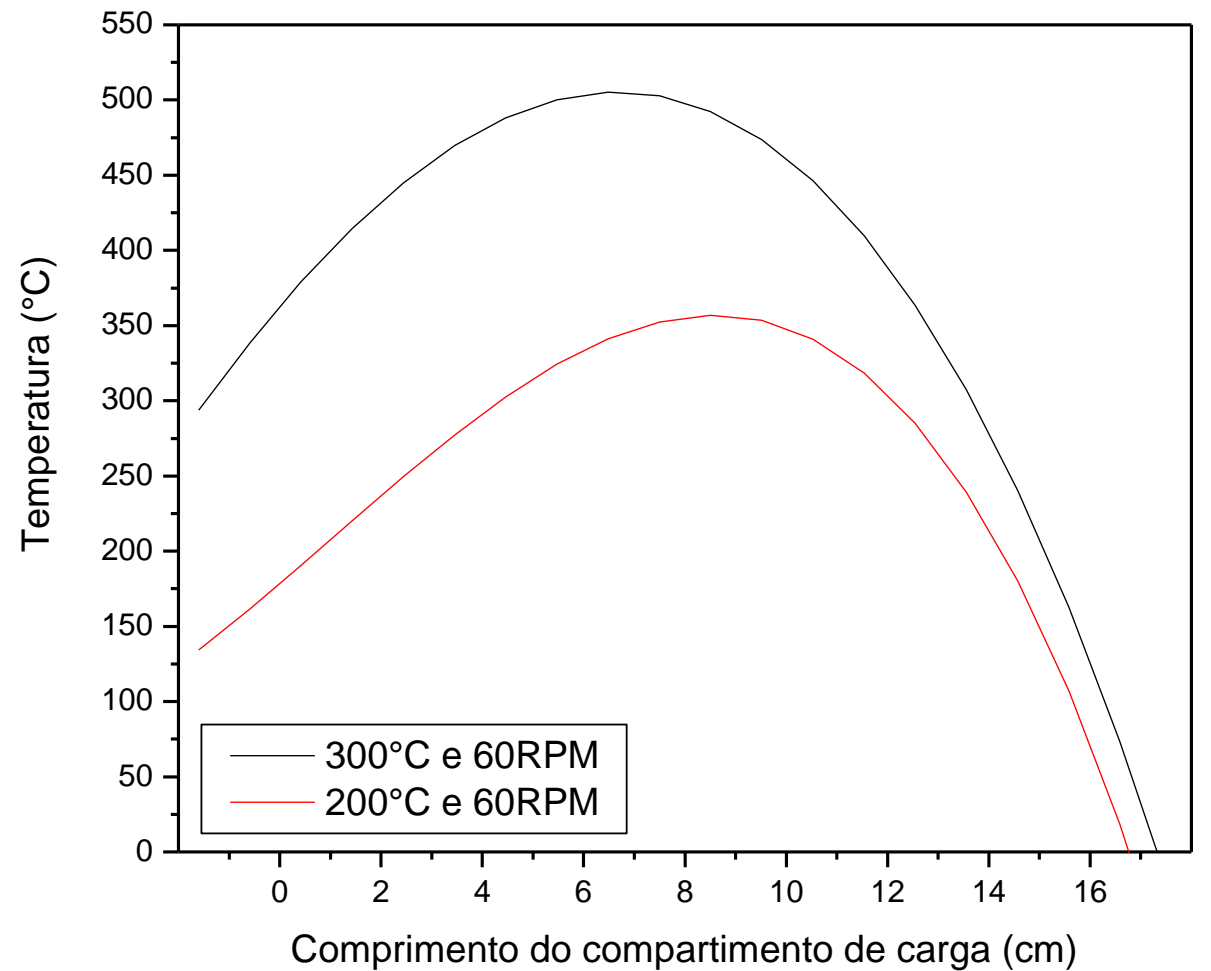

Figura 10. Perfil do forno dinâmico. 


\subsection{Simulações de rotação}

Nas simulações de rotação do VSB-30 o forno ficará fixo em cima de um disco utilizado em voo, deslocado poucos centímetros do centro de rotação (Figura 16). Todas as configurações do ensaio serão iguais a da Operação Maracati II [16 e 17].

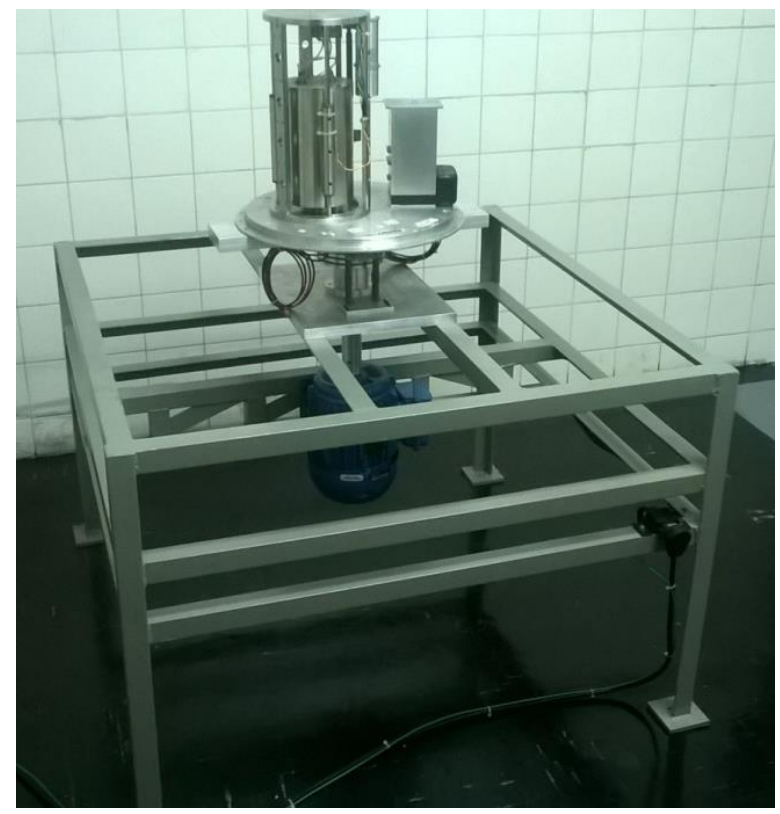

Figura 11. Equipamento para simulação de rotações

O balanceamento do disco foi realizado em software devido à complexidade da geometria. Assim, a massa contra peso utilizada será 2,085 kg.

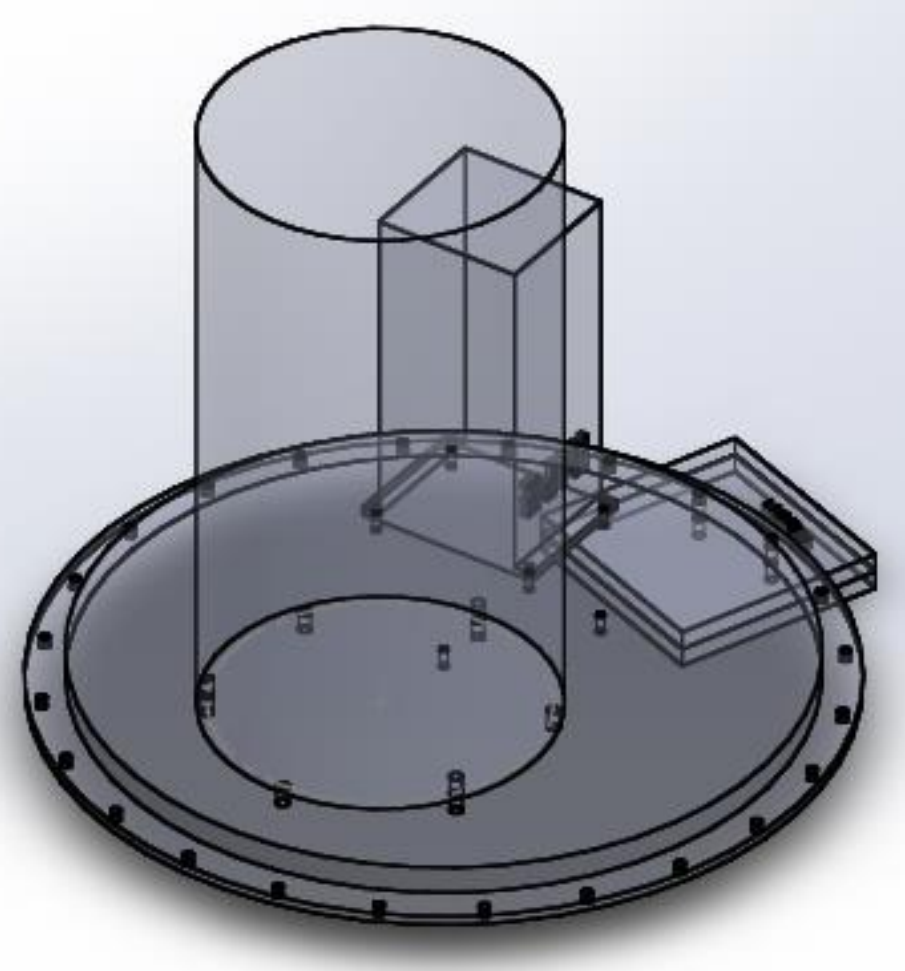

Figura 12. Fixação do forno para simulação da rotação. 


\section{CONCLUSÃO}

Com as metas elaboradas no início do projeto alcançadas, o desenvolvimento deste equipamento chegou ao fim, sendo atualmente utilizado no estudo de ligas metálicas em condições de altas acelerações. Além disso, a centrífuga irá auxiliar o LAS/INPE no estudo dos mecanismos da solidificação de materiais em macrogravidade. Fazendo do laboratório um dos poucos no Brasil a dispor dessa tecnologia.

\section{Agradecimentos}

Os autores são gratos ao CNPq e a Capes pelo suporte financeiro.

\section{REFERÊNCIAS}

1 AN, C. Y.; RUSSO, L. C.; RIBEIRO, M. F.; BANDEIRA, I. N. A Low Cost Centrifuge for Materials Processing in High Gravity. No: Centrifugal Materials Processing. New York: Plenum Press, 1997.

2 KURZ, W.; FISHER, D. J. Fundamentals of solidification. 3. ed. [S.I.]: Trans Tech Publications Ltd, 316 p. 1992.

3 CHIRITA, G.; SOARES, D.; SILVA, F. S. Advantages of the centrifugal casting technique for the production of structural components with $\mathrm{Al}-\mathrm{Si}$ alloys. Materials and Design. v. 29, p. 20 -27. 2008.

4 LIMIN, J.; ZHIMIN, L.; DAMING, X.; JINGJIE, G. Effects of Centrifugal Forces and Casting Modulus on Structures and Mechanical Properties of Ti-6Al-4V Alloy. Rare Metal Materials and Engineering. v. 45(3): 0581-0587. 2016.

5 FREITAS, F. E.; TOLEDO, R. C.; POLI, A. K. S.; AN, C. Y.; BANDEIRA, I. N. The influence of high gravity in $\mathrm{PbSn}$ eutectic alloy. No: 21 Congresso Brasileiro de Engenharia e Ciência dos Materiais CBECiMat, Cuiabá. Anais do 21 Congresso Brasileiro de Engenharia e Ciência dos Materiais CBECiMat. p. 6551-6557, 2014.

6 BÁRCZY, P.; SOLYÓM, J.; REGEL, L.; Solidification of Al-AI3Ni(Cu) eutectics at high gravity. Journal of Crystal Growth. v. 119, p. 160-166. 1992.

7 NUSSENZVEIG, H. M. Curso de Física Básica. 4a edição. São Paulo. Edgard Blücher. ISBN 58-212-0298-9. 2002.

8 TENÓRIO, P. I. G.; TOLEDO, R. C.; FREITAS, F. E.; FUMACHI, E. F.; BANDEIRA, I. N.; AN, C. Y. Desenvolvimento de uma centrífuga para solidificação de ligas eutéticas em macrogravidade. No: VI Workshop em Engenharia e Tecnologia Espaciais, São José dos Campos/SP. Anais do VI Workshop em Engenharia e Tecnologia Espaciais, 2015.

9 IAE - INSTITUTO DE AERONÁUTICA E ESPAÇO. VSB-30. Disponível em: http://www.iae.cta.br/site/page/view/pt.vsb30.html. Acesso: 06/07/2015.

10 AEB - AGÊNCIA ESPACIAL BRASILEIRA. Operação Maracati II. Disponível em: http://microgravidade.aeb.gov.br/index.php/missoes/maracati-ii. Acesso: 21/04/2017.

11 AN, C. Y.; BOSCHETTI, C.; RIBEIRO, M. F.; TOLEDO, R. C.; FREITAS, F. E.; CASTILHO, F. F.; BANDEIRA, I. N. Solidification furnace for microgravity experiments on sounding rockets. Journal of Aerospace Technology and Management (Online). v. 4. p. 237-240. 2012.

12 HALLIDAY, D.; RESNICK, R.; WALKER, J. Fundamentos da Física, Vol. 1, 8ª Edição, LTC, 2009. 
13 FERRO, R. M.; FERREIRA, W. G.; CALENZANI, A. F. G. Estado da Arte: Análise Dinâmica de Estruturas de Aço Suportes de Máquinas Rotativas. No: XI Simpósio de Mecânica Computacional (SIMMEC) / II Encontro Mineiro de Modelagem Computacional, Juiz de Fora. Anais do SIMMEC/EMMCOMP 2014, 2014.

14 WEG. W22 Motor Elétrico Trifásico - Catálogo Técnico Mercado Brasileiro. WEG, 2016.

15 WEG. Manual do Usuário, Inversor de Frequência CFW-08. WEG, 213 p. 2009.

16 BANDEIRA, I. N.; AN, C. Y.; BOSCHETTI, C.; RIBEIRO, M. F.; PEIXOTO, J. C.; SILVA, S.A. Relatório Final Operação Cumã: Experimento Pem05 - Forno Multisuário Para Solidificação (Formu-S). Relatório de Pesquisa. 32p. Instituto Nacional de Pesquisas Espaciais, São José dos Campos, 2013

17 AN, C.Y.; BANDEIRA, I. N.; BOSCHETTI, C.; RIBEIRO, M. F.; PEIXOTO, J. C.; TOLEDO, R. C. Relatório de Participação na Operação maracati - 2. Relatório de Pesquisa. 15p. Instituto Nacional de Pesquisas Espaciais, São José dos Campos, 2013.

18 HIBBELER, R. C. Resistência dos materiais. $7^{a}$ edição. São Paulo. Person Pretince Hall, 2009. 